\title{
Predictive Value of Heidelberg Retina Tomograph Parameters for the Development of Glaucoma in the European Glaucoma Prevention Study
}

\section{STEFANO MIGLIOR, THIERRY ZEYEN, ESTHER M. HOFFMANN, VALTER TORRI, ELIANA RULLI, IRENE FLORIANI, DAVIDE POLI, SHAKHSANAM ALIYEVA, JOSÉ CUNHA-VAZ, AND NORBERT PFEIFFER}

- PURPOSE: To determine whether baseline Heidelberg Retina Tomograph (HRT) measurements of the optic disc are associated with the development of open-angle glaucoma $(\mathrm{OAG})$ in individuals with ocular hypertension in the European Glaucoma Prevention Study (EGPS).

- DESIGN: Retrospective analysis of a prospective, randomized, multicenter, double-masked, controlled clinical trial.

- METHODS: There were 489 participants in the HRT Ancillary Study to the EGPS. Each baseline HRT parameter was assessed in univariate and multivariate proportional hazards models to determine its association with the development of OAG. Proportional hazards models were used to identify HRT variables that predicted which participants in the EGPS had developed OAG. Development of OAG was based on visual field and/or optic disc changes.

- RESULTS: At a median follow-up time of about 5 years, 61 participants developed OAG. In multivariate analyses, adjusting for randomization arm, age, baseline IOP, central corneal thickness, pattern standard deviation, and HRT disc area, the following HRT parameters were associated with the development of OAG: the "outside normal limits" classification of the Frederick Mikelberg (FSM) discriminant function (hazard ratio [HR] 2.51, 95\% confidence interval [CI]: 1.45-4.35), larger mean cup depth (HR 1.64, 95\% CI: 1.21-2.23), cup-to-disc area ratio (HR 1.43, 95\% CI: 1.14-1.80), linear cup-to-disc ratio (HR 1.43, 95\% CI: $1.13-1.80$ ), cup area (HR 1.33, 95\% CI: 1.08-1.64), smaller rim area (HR 1.33, 95\% CI: $1.07-1.64)$, larger cup volume (HR $1.30,95 \% \mathrm{CI}$ : 1.05-1.61), smaller rim volume (HR 1.25, 95\% CI: 1.01-1.54), larger maximum cup depth (HR 1.18, 95\% CI: 1.01-1.36), and cup shape measure (HR 1.18, 95\% CI: $1.01-1.36)$.

Accepted for publication Oct 27, 2014.

From the Department of Ophthalmology, University of Milan Bicocca, Policlinico di Monza, Monza, Italy (S.M.); Leuven University Hospitals, Leuven, Belgium (T.Z.); Department of Ophthalmology, University Medical Center Mainz, Mainz, Germany (E.M.H., S.A., N.P.); IRCCS Istituto di Ricerche Farmacologiche "Mario Negri," Milan, Italy (V.T., E.R., I.F., D.P.); and Department of Ophthalmology, Coimbra University Hospital, Coimbra, Portugal (J.C.-V.).

Inquiries to Stefano Miglior, Universita' di Milano Bicocca, Policlinico di Monza, Via Amati 111, 20052, Monza (Mi), Italy; e-mail: stefano, miglior@unimib.it
- CONCLUSIONS: Several baseline HRT parameters, alone or in combination with baseline clinical and demographic factors, were significantly associated with the development of OAG among the EGPS participants. (Am J Ophthalmol 2015;159:265-276. (C) 2015 by Elsevier Inc. All rights reserved.)

$\mathrm{P}$ REVENTION OF BLINDNESS FROM GLAUCOMA remains one of the major goals in ophthalmology. At present the therapeutic strategies are mainly based on a medical or a surgical approach aimed at decreasing intraocular pressure (IOP). Ocular hypertension (OHT) has been recognized as the most important risk factor for the development of open-angle glaucoma $(\mathrm{OAG})^{1-6}$ and, as of today, is the only factor that can be significantly influenced by medication or surgery. Among other risk factors that are deemed important in the genesis of the disease, such as age, race, family history, and low diastolic perfusion pressure, ${ }^{3-9}$ only the latter can hypothetically benefit from a multidisciplinary therapeutic approach.

A strategy aimed at preventing the onset of $O A G$ among patients with $\mathrm{OHT}^{10}$ is best based on the identification of predictive factors. Older age, higher IOP, larger vertical or horizontal cup-to-disc $(\mathrm{C} / \mathrm{D})$ ratio, greater pattern standard deviation (PSD), and thinner central corneal thickness (CCT) at baseline have been found to be predictors for the onset of OAG in the Ocular Hypertension Treatment Study (OHTS $)^{11}$ and in the European Glaucoma Prevention Study (EGPS). ${ }^{12}$ By merging the datasets of the OHTS and the EGPS it has been possible to provide a risk calculator for the development of primary open-angle glaucoma (POAG) within 5 years, which may estimate the individual risk profile and be helpful to the clinician in establishing the management of OHT in each specific patient. ${ }^{13,14}$

Both the OHTS and the EGPS have conducted an ancillary study in a subset of the original sample based on the use of the Heidelberg Retina Tomograph (HRT) in order to assess whether HRT results could be predictive for the development of OAG. From the introduction of the first dedicated HRT confocal scanning laser ophthalmoscope (CSLO) in 1989 for disc imaging and topography, ${ }^{15}$ the overall agreement based on the results of several studies is that HRT may identify 
early signs of structural glaucomatous damage. ${ }^{16-33}$ The HRT Ancillary Study of the OHTS has confirmed these observations, suggesting that the clinical use of HRT in OHT patients may predict the development of POAG. ${ }^{34-36}$

This report describes the HRT predictive parameters for development of OAG among the OHT patients enrolled in the EGPS. ${ }^{37}$ Given the similarities between the protocols for the OHTS $^{34}$ and the EGPS, ${ }^{37}$ our results may add to the refinement of a more accurate identification of OHT patients who may warrant treatment with ocular hypotensive medications, and are the ground for the ongoing OHTS-EGPS HRT Collaborative Study.

\section{METHODS}

THE EGPS WAS A MULTICENTER RANDOMIZED, DOUBLEmasked, placebo-controlled clinical trial. ${ }^{38}$ Since it was planned in 1995, it started thereafter, and it was not ongoing by December 2007, the EGPS is not registered either at "clinicaltrials.gov" or at "controlled-trials.com." The design and methods of the EGPS were previously described $^{39}$ and are summarized as follows. This HRT Ancillary Study to the EGPS is a retrospective analysis of the original prospective clinical trial.

The protocol was approved by the ethical review committee of each participating clinic, and each patient gave his or her informed consent to be enrolled in the study. In brief, 1081 individuals with IOP $\geq 22 \mathrm{~mm} \mathrm{Hg}$ in at least 1 eye and no evidence of glaucomatous damage were randomized to either dorzolamide or placebo. Four patients who were found to have glaucoma at the time of randomization were discontinued from the study and excluded from further analyses, leaving a total of 1077 patients. The primary outcome was the development of a reproducible visual field change from baseline, or a clinically detectable optic disc change from baseline as determined by 2 of 3 independent evaluators. ${ }^{40} \mathrm{Vi}$ sual field (VF) and optic disc changes were identified by masked certified evaluators at the central coordinating center, and the confirmation that the endpoint was attributable to OAG was determined after masked review of the clinical charts. Both visual field and optic disc photographs were performed every 6 months. Worsening of visual field was reached when at least 1 of the following criteria was met: (1) $\geq 3$ horizontally or vertically adjacent points that differ $\geq 5 \mathrm{~dB}$ from baseline, $(2) \geq 2$ horizontally or vertically adjacent points that differ $\geq 10 \mathrm{~dB}$ from baseline, (3) difference of $\geq 10$ $\mathrm{dB}$ across nasal horizontal meridian at $\geq 2$ adjacent points. The loss had to be not attributable to other pathologies.

The sensitivity loss was defined relative to the baseline (normal) values of each patient. The superior and inferior rows of the physiologic blind spot were excluded from the field evaluation. To meet the criteria that defined the occurrence of visual field endpoint, the patient had to repeat the VF testing within 30 days. If the defect was confirmed in the same test locations, the patient had to repeat a third VF test. If the defect was confirmed again, the VF endpoint was considered as met (the $3 \mathrm{VF}$ results had to be consecutive). In case of questionable worsening the patient continued the study and repeated the VF test at the next follow-up visit. Worsening of the optic disc was defined as a visually recognizable (on stereophotographs) narrowing of the neuroretinal rim area (localized or diffuse) not attributable to photographic artifacts. This was detected by comparing follow-up stereoscopic optic disc slides with baseline stereoscopic optic disc slides. An optic disc endpoint was reached when 2 out of 3 optic disc reading centers independently determined worsening. If the worsening appeared questionable, the patient continued in the study and pictures were taken again at the next follow-up visit.

A detailed collection of clinical data was obtained from each participant by self-report. This information included ocular and medical history and current use of medication. Medical history was obtained by self-report from the participant.

Baseline demographic and clinical information were collected for each participant prior to randomization. The baseline and follow-up visits included assessment of refraction and visual acuity using the standard procedures at each given office; Goldmann applanation tonometry performed and recorded by a single investigator between 8:00 and 11:00 AM (ie, at least 1 but not more than 3 hours after the last dose of study medication); complete ophthalmologic examination including gonioscopy, automated static perimetry with a Humphrey (Carl Zeiss Meditec, Dublin, California, USA) or Octopus (Haag-Streit AG, Koeniz-Berne, Switzerland) instrument using a central 30-degree program with threshold double-crossing strategy; and color slide stereophotography of the optic disc. Myopia was defined as a spherical equivalent of -1.0 diopter (D) or more. Horizontal and vertical $\mathrm{C} / \mathrm{D}$ ratios by contour were estimated visually from stereoscopic optic disc photographs by masked certified readers. $\mathrm{C} / \mathrm{D}$ ratio asymmetry was calculated by subtracting the $C / D$ ratio value of 1 eye from that of the other eye.

In the EGPS, 241 out of 1077 patients underwent Octopus visual field evaluations, which calculate "loss variance" instead of "pattern standard deviation." Therefore, the indices of the Octopus were converted to the indices of the Humphrey system. One approach could have been the algorithms reported by Zeyen and associates, ${ }^{41}$ but these were derived from glaucomatous visual fields. Based on personal information (Heijl A, and Johnson C, oral communication, September 1, 2005), the mean defect, loss variance, and corrected loss variance of the Octopus system were converted to the mean deviation (MD), PSD, and corrected pattern standard deviation (CPSD) of the Humphrey visual field testing. This was performed by changing the sign in front of the value of mean defect and by calculating the square root of the loss variance and corrected loss variance, respectively. ${ }^{12}$

Although not included in the original protocol of the study, CCT measurements were taken during the trial in 
a large sample of the patients: 429 in the dorzolamide group $(80.0 \%)$ and 425 in the placebo group $(78.5 \%) .{ }^{42}$ CCT was performed using the same pachymeter employed in the OHTS (DGH-500 Pachette; DGH Technologies, Exton, Pennsylvania, USA) ${ }^{43}$ following a standard procedure in all the centers, which included the use of topical anesthesia and the acquisition of 5 measurements in both eyes. The average of the mean of the 5 measurements of the 2 eyes was considered for analysis. When only 1 eye was included in the study, the mean of the 5 measurements of that eye was considered for the analysis. This measurement was begun in 2002, 3 years after the randomization of the last patient enrolled in the study.

In the EGPS, a total of 106 out of 1077 patients developed OAG over the course of the trial. However, among the patients who discontinued the study and were followed according to the same protocol until the end of the study (293 patients), 14 developed a visual field (6) or optic disc (8) endpoint after the discontinuation for a total of 120 patients who developed $O A G$, regardless of the time that OAG occurred.

In this publication, glaucoma is always referred to as OAG for consistency throughout the manuscript. It is noted that the EGPS allowed for the inclusion of patients affected by pigment dispersion syndrome (PDS) or pseudoexfoliation syndrome (PEX), which may lead to secondary OAG.

- HEIDELBERG RETINA TOMOGRAPH: The Heidelberg Retina Tomograph (HRT; Heidelberg Engineering GmbH, Heidelberg, Germany) is a confocal scanning laser device that uses a 670-nm diode laser to obtain 2- and 3dimensional images of the optic nerve head and the peripapillary retina. ${ }^{15}$ HRT examinations within the EGPS started at the baseline examination. The HRT images were obtained every 6 months or at least annually. In all eyes, 10-degree HRT images were acquired at baseline and throughout the study using the older hardware version (HRT "classic"). A topographic image is built from a series of 32 consecutive optical sections, each consisting of $256 \times 256$ pixels. A mean image is generated by 3 consecutive scans. A minimum of 2 but preferably 3 images were obtained, and a mean image was created for each eye. Keratometry measurements were used to correct for magnification error. For analysis purposes, all HRT data were analyzed in software version 3.0 (Heyex platform).

Assessment of image quality was performed by means of the automatic quality control "Auto QC" feature of the software according to the following recommended criteria to reject images: underexposure, severe overexposure, focus greater than or equal to 75 diopters, scan depth too small, scan depth too high (equal to or greater than $1 \mathrm{~mm}$ ), standard deviation greater than $50 \mu \mathrm{m}$ (mean pixel height standard deviation). The optic disc margin, defined as the inner margin of the scleral ring, was outlined on the mean topography image by 1 experienced examiner. Each outline of the optic disc was reviewed for accurate placement by a second experienced examiner (with differences of opinion resolved by consensus). The reference plane was automatically determined at $50 \mu \mathrm{m}$ posterior to the mean retinal height between 350 degrees and 356 degrees (papillomacular bundle) along the contour line.

Automatic HRT image quality assessment and drawing of the contour line took place at the EGPS HRT Reading Center at the Department of Ophthalmology, University Medical Center of Mainz, Germany. A second independent image quality control step was performed at the OHTS CSLO Reading Center, University of California, San Diego (San Diego, California, USA), with the goal of including as many "good-quality" scans as possible, using the same review criteria that had been used for the OHTS CSLO Ancillary Study. ${ }^{34-36}$

The following stereometric parameters and multidiscriminant functions were calculated and evaluated: disc area; cup area; rim area; cup volume; rim volume; $\mathrm{C} / \mathrm{D}$ area; linear $\mathrm{C} / \mathrm{D}$; mean cup depth; maximum cup depth; cup shape measure; height variation contour (HVC); retinal nerve fiber layer (RNFL) cross-sectional area; mean RNFL thickness; reference plane height; standard deviation; Frederick Mikelberg's discriminant function (FSM), which corresponds to the "HRT classification"; Reinhard Burk's discriminant function (RB); Moorfields regression analysis (MRA); and the Glaucoma Probability Score (GPS). The FSM uses a formula that takes into account rim volume, cup shape measure, and height variation contour, adjusting for age. ${ }^{16}$ The RB uses the same parameters as the FSM, but weights them differently. ${ }^{25}$ Both discriminant analyses classify the image as being normal or outside normal limits. MRA compares global and sectorial rim area (after adjusting for disc area) to a normative database. ${ }^{21}$ MRA is classified as "within normal limits" if a given rim area is equal to or greater than confidence interval at 95\% (95\% CI), "borderline" if it is between 95\% and 99.9\% CI, and "outside normal limits" if it is $>99.9 \%$ CI. GPS uses 2 measurements of the peripapillary retinal nerve fiber layer shape (horizontal and vertical RNFL curvature) and 3 measurements of the optic nerve head shape (cup size, cup depth, and rim steepness) for input into a vector machine-learning classifier that estimates the probability of having damage consistent with glaucoma. ${ }^{24}$ Both MRA and GPS classify the image as being within normal limits, borderline, or outside normal limits. For the sake of the analysis, results classified as being "borderline" have been considered as being "within normal limits."

- STATISTICAL ANALYSIS: When both eyes were included in the study (366 patients), ${ }^{13}$ for some eye-specific variables, the mean for each eye (eg, the mean of the 2 IOP or the 5 CCT measurements) was calculated and then these 2 values were averaged to determine the baseline predictive factor. For other eye-specific variables, the second obtained measurement (eg, the second baseline visual field test) or the only available measurement (eg, the $\mathrm{C} / \mathrm{D}$ ratios) was 
used and then the values of the 2 eyes were averaged to determine the baseline predictive factor. When 1 eye only was included in the study (123 patients), only the data from the included eye were used. When both eyes were included in the study, the mean of the 2 eyes was calculated for each HRT parameter. In the case of FSM, RB, MRA, and GPS, if 1 of the 2 included eyes was outside normal limits then the classification for study purposes was "outside normal limits." When only 1 eye was included in the study, only the data from the included eye were used.

Cox proportional hazards models as implemented in the PHREG program in the SAS statistical software (SAS Institute Inc, Cary, North Carolina, USA) were used to estimate and test factors for their association with the development of OAG. The analysis sample for the proportional hazards models consisted of 61 randomized participants who developed POAG and 428 randomized patients who did not develop OAG before the end of the trial (September 2003). Median participant follow-up was 59.5 months, or about 5 years. Proportional hazard models were used for univariate and multivariate analysis. Variables that were found to be associated with OAG development in the univariate analysis $(P<.1)$ were considered for the multivariate analysis. Multivariate Cox proportional hazards models were evaluated in the entire study sample using the treated arm as stratification variables. Two multivariate analyses were performed for each single HRT parameter that was associated with OAG development in univariate analysis, the former also including in the model age, CCT, baseline mean IOP, and PSD, which are the baseline predictive factors for the development of OAG found in the EGPS, ${ }^{12}$ and the latter including in the model the same baseline factors and HRT disc area, given the previously reported high correlation between several HRT parameters and HRT disc area. ${ }^{37,44-47}$ Vertical C/D ratio, which is also a significant predictive factor for OAG in the EGPS, ${ }^{12}$ was not included in any analysis given the consistency of HRT and ophthalmoscopic evaluations, obviously performed on the same anatomic structure (the optic disc). ${ }^{37}$ With a stratified Cox model, a proportional hazard structure is assumed to hold within each stratum. The relative effect of each predictor is assumed the same across strata, unless there is a significant strata-bycovariate interaction, which implies that the effect of the particular covariate differs within strata. One disadvantage of using a stratified model is that an effect of the stratification covariate cannot be estimated in the model, at least in the sense of a coefficient estimate. Since we were not interested in estimating the effect of treatment, but only in taking it into account when estimating the effect of other variables, the stratified model seemed the most appropriate method to be used, as also adopted by others (OHTS). ${ }^{11}$ Results are expressed as hazard ratios (HRs) with 95\% CIs Statistical significance was defined as $P<.05$ for a bilateral test.

A 2 -sided log-rank test was performed at the $5 \%$ significance level. The primary variable was the length of time to develop glaucomatous damage, that is, the time from
TABLE 1. Baseline Demographic Characteristics by OpenAngle Glaucoma Status (Average of the Eyes)

\begin{tabular}{|c|c|c|c|}
\hline \multirow[b]{2}{*}{ Characteristic } & \multicolumn{3}{|c|}{ Number (\%) } \\
\hline & Normal & OAG Endpoint & All \\
\hline \multicolumn{4}{|l|}{ Sex } \\
\hline Female & $232(87.55)$ & $33(12.45)$ & 265 (100) \\
\hline Male & $196(87.50)$ & $28(12.50)$ & $224(100)$ \\
\hline \multicolumn{4}{|l|}{ High blood pressure } \\
\hline No & $280(88.89)$ & $35(11.11)$ & $315(100)$ \\
\hline Yes & $148(85.06)$ & $26(14.94)$ & $174(100)$ \\
\hline \multicolumn{4}{|c|}{ Cardiovascular diseases } \\
\hline No & 372 (88.78) & $47(11.22)$ & 419 (100) \\
\hline Yes & $56(80.00)$ & $14(20.00)$ & 70 (100) \\
\hline \multicolumn{4}{|l|}{ Diabetes } \\
\hline No & $399(87.31)$ & 58 (12.69) & $457(100)$ \\
\hline Yes & $29(90.63)$ & $3(9.38)$ & $32(100)$ \\
\hline \multicolumn{4}{|l|}{ Diuretics } \\
\hline No & $380(88.99)$ & 47 (11.01) & $427(100)$ \\
\hline Yes & 48 (77.42) & $14(22.58)$ & $62(100)$ \\
\hline \multicolumn{4}{|l|}{ ACE inhibitors } \\
\hline No & $321(88.43)$ & $42(11.57)$ & 363 \\
\hline Yes & $107(84.92)$ & $19(15.08)$ & 126 \\
\hline \multicolumn{4}{|c|}{ Calcium channel blockers } \\
\hline No & $366(88.41)$ & 48 (11.59) & $414(100)$ \\
\hline Yes & $62(82.67)$ & $13(17.33)$ & 75 (100) \\
\hline \multicolumn{4}{|l|}{ Beta blockers } \\
\hline No & $359(87.60)$ & 48 (12.40) & 387 (100) \\
\hline Yes & $89(87.25)$ & $13(12.75)$ & $102(100)$ \\
\hline \multicolumn{4}{|l|}{ FSM } \\
\hline Normal $^{a}$ & 337 (90.59) & 35 (9.41) & 372 (100) \\
\hline Abnormal $^{b}$ & $91(77.78)$ & $26(22.22)$ & 117 (100) \\
\hline \multicolumn{4}{|l|}{ GPS } \\
\hline Normal $^{a}$ & 349 (88.35) & 46 (11.65) & 395 (100) \\
\hline Abnormal $^{b}$ & 63 (82.89) & $13(17.11)$ & 76 (100) \\
\hline \multicolumn{4}{|l|}{ MRA } \\
\hline Normal $^{a}$ & 394 (88.74) & 50 (11.26) & 444 (100) \\
\hline Abnormal $^{b}$ & $34(75.56)$ & $11(24.44)$ & 45 (100) \\
\hline \multicolumn{4}{|c|}{$\begin{array}{l}\text { ACE }=\text { angiotensin-converting enzyme; FSM = Frederick } \\
\text { Mikelberg discriminant function; GPS = Glaucoma Probability } \\
\text { Score; MRA = Moorfields regression analysis; OAG = open- } \\
\text { angle glaucoma } \\
{ }^{a} \text { Within normal limits + borderline. } \\
{ }^{b} \text { Outside normal limits. }\end{array}$} \\
\hline
\end{tabular}

randomization to first confirmed occurrence of a worsened visual field or a worsened optic disc. The univariate difference between a baseline abnormal or normal HRT examination with respect to the primary variable was assessed using survival analysis.

\section{RESULTS}

OF THE 1077 PARTICIPANTS IN THE EGPS STUDY, 489 (45.4\%) were included in the HRT study, accounting for 4052 
TABLE 2. Baseline Demographic and Ocular Characteristics by Open-Angle Glaucoma Status (Average of the Eyes)

\begin{tabular}{|c|c|c|c|c|c|c|}
\hline \multirow[b]{2}{*}{ Characteristic } & \multicolumn{2}{|c|}{ Normal } & \multicolumn{2}{|c|}{ OAG Endpoint } & \multicolumn{2}{|c|}{ All } \\
\hline & Sample Size & Mean (SD) & Sample Size & Mean (SD) & Sample Size & Mean (SD) \\
\hline Age & 428 & $56.70(9.57)$ & 61 & $61.74(8.87)$ & 489 & $57.33(9.62)$ \\
\hline Baseline IOP & 428 & $23.62(1.52)$ & 61 & $24.04(1.78)$ & 489 & $23.68(1.56)$ \\
\hline Refraction & 428 & $0.21(1.89)$ & 61 & $0.56(1.80)$ & 489 & $0.26(1.88)$ \\
\hline MD & 428 & $0.44(1.44)$ & 61 & $0.91(1.33)$ & 489 & $0.50(1.43)$ \\
\hline PSD & 428 & $1.97(0.58)$ & 61 & $2.07(0.56)$ & 489 & $1.98(0.58)$ \\
\hline CPSD & 428 & $1.05(0.66)$ & 61 & $1.17(0.59)$ & 489 & $1.07(0.66)$ \\
\hline Best-corrected visual acuity & 428 & $-0.02(0.06)$ & 61 & $-0.02(0.04)$ & 489 & $-0.02(0.06)$ \\
\hline CCT & 351 & $578.82(36.88)$ & 57 & $560.87(29.03)$ & 408 & $576.31(36.39)$ \\
\hline Vertical C/D & 428 & $0.34(0.14)$ & 61 & $0.40(0.13)$ & 489 & $0.35(0.14)$ \\
\hline Vertical C/D asymmetry & 428 & $0.05(0.06)$ & 61 & $0.09(0.08)$ & 489 & $0.05(0.07)$ \\
\hline Keratometry & 428 & $7.68(0.17)$ & 61 & $7.69(0.13)$ & 489 & $7.68(0.17)$ \\
\hline \multicolumn{7}{|l|}{ HRT parameters } \\
\hline Disc area & 428 & $2.05(0.42)$ & 61 & $2.02(0.40)$ & 489 & $2.04(0.42)$ \\
\hline Cup area & 428 & $0.51(0.38)$ & 61 & $0.62(0.38)$ & 489 & $0.53(0.38)$ \\
\hline Rim area & 428 & $1.53(0.29)$ & 61 & $1.40(0.34)$ & 489 & $1.52(0.30)$ \\
\hline Cup volume & 428 & $0.12(0.13)$ & 61 & $0.16(0.15)$ & 489 & $0.13(0.14)$ \\
\hline Rim volume & 428 & $0.41(0.15)$ & 61 & $0.36(0.14)$ & 489 & $0.41(0.15)$ \\
\hline C/D area & 428 & $0.24(0.14)$ & 61 & $0.29(0.14)$ & 489 & $0.25(0.14)$ \\
\hline C/D linear & 428 & $0.45(0.16)$ & 61 & $0.52(0.15)$ & 489 & $0.46(0.16)$ \\
\hline Cup depth & 428 & $0.21(0.09)$ & 61 & $0.25(0.10)$ & 489 & $0.22(0.09)$ \\
\hline Maximum cup depth & 428 & $0.57(0.20)$ & 61 & $0.63(0.21)$ & 489 & $0.58(0.20)$ \\
\hline Cup shape measurement & 428 & $-0.17(0.07)$ & 61 & $-0.15(0.07)$ & 489 & $-0.17(0.07)$ \\
\hline HVC & 428 & $0.39(0.09)$ & 61 & $0.39(0.09)$ & 489 & $0.39(0.09)$ \\
\hline RNFL thickness & 428 & $0.24(0.06)$ & 61 & $0.23(0.07)$ & 489 & $0.24(0.06)$ \\
\hline RNFL CSA & 428 & $1.22(0.33)$ & 61 & $1.14(0.33)$ & 489 & $1.21(0.33)$ \\
\hline Reference height & 428 & $0.30(0.09)$ & 61 & $0.30(0.09)$ & 489 & $0.30(0.09)$ \\
\hline SD & 428 & $17.89(7.14)$ & 61 & $18.79(7.20)$ & 489 & $18.00(7.14)$ \\
\hline RB formula & 428 & $1.29(0.84)$ & 61 & $1.15(0.83)$ & 489 & $1.27(0.84)$ \\
\hline
\end{tabular}

good-quality HRT images taken during the study. Images were taken in both eyes in 366 participants whereas they were taken in 1 eye only (the same included in the clinical trial) in 123 participants, for a total of 855 eyes. As the EGPS clinical trial began before the availability of the confocal scanning laser ophthalmoscope at all the sites involved in the EGPS HRT substudy, not all participants completed their imaging at the EGPS baseline visit. Of the 489 participants with good-quality images included in this study, 177 (36.2\%) had images obtained at the baseline visit, $76(15.5 \%)$ at the 6 - or 12 -month visit, 122 (24.9\%) at the 18 - or 24 -month visit, $44(9 \%)$ at the 30 - or 36 month visit, and 70 (14.3\%) later. Among the 312 participants who were not evaluated by means of HRT at baseline, the mean $( \pm S D)$ time between randomization and first HRT examination was $2.06 \pm 1.3$ years in 267 participants not developing $\mathrm{OAG}$, and $1.59 \pm 0.56$ years in 45 participants who developed OAG.

Demographic characteristics of the subjects participating and nonparticipating in the HRT study have been previously published. ${ }^{37}$ Table 1 and Table 2 report those of the 489 OHT patients by OAG status. At a median follow-up of 59.5 months 61 patients developed OAG, defined by an optic disc endpoint in 27 and by a visual field endpoint in 34 cases. Fifty-two endpoints were reached during the trial and 9 endpoints were reached after discontinuation of the patient from the clinical trial.

The multivariate analysis of the baseline predictive factors previously reported by the EGPS ${ }^{12}$ in this subset of the original population shows that the HRs (95\% CI) for age, IOP, CCT, vertical C/D ratio, and PSD are 1.52 (1.05-2.19), 1.10 (0.93-1.30), 1.71 (1.21-2.41), 1.88 (1.38-2.58), and 1.53 (0.92-2.56), respectively. Univariate HRs with 95\% CIs are reported for each putative HRT predictive factor for the development of OAG in Table 3. In univariate analyses, baseline HRT parameters significantly predictive of the development of OAG were rim area, cup area, rim volume, $\mathrm{C} / \mathrm{D}$ area ratio, $\mathrm{C} / \mathrm{D}$ linear ratio, cup depth, maximum cup depth, cup shape measure, FSM function outside normal limits, and MRA result outside normal limits. 
TABLE 3. Univariate Hazard Ratios and 95\% Confidence Intervals for the Development of Open-Angle Glaucoma (Average of the Eyes)

\begin{tabular}{lcl}
\hline \multicolumn{1}{c}{ HRT Parameter } & HR $(95 \% \mathrm{Cl})$ & \multicolumn{1}{c}{$P$} \\
\hline Disc area (per 0.4 $\mathrm{mm}^{2}$ greater) & $0.95(0.75-1.21)$ & .70 \\
Cup area (per $0.3 \mathrm{~mm}^{2}$ greater) & $1.12(1.00-1.25)$ & .057 \\
Rim area (per $0.2 \mathrm{~mm}^{2}$ greater) & $0.75(0.62-0.90)$ & .002 \\
Cup volume (per $0.1 \mathrm{~mm}^{2}$ greater) & $1.16(1.00-1.35)$ & .045 \\
Rim volume (per $0.1 \mathrm{~mm}^{3}$ greater) & $0.79(0.65-0.96)$ & .016 \\
C/D area (per 0.1 greater) & $1.29(1.09-1.53)$ & .003 \\
C/D linear (per 0.1 greater) & $1.28(1.08-1.52)$ & .005 \\
Cup depth (per 0.1 mm greater) & $1.46(1.14-1.88)$ & .003 \\
Max cup depth (per 0.1 mm greater) & $1.14(1.01-1.30)$ & .042 \\
Cup shape measure (per 0.1 greater) & $1.75(1.19-2.57)$ & .004 \\
HVC (per 0.1 greater) & $0.97(0.73-1.28)$ & .82 \\
RNFL thickness (per 0.1 greater) & $0.73(0.49-1.09)$ & .12 \\
RNFL CSA (per 0.3 mm ${ }^{2}$ greater) & $0.94(0.86-1.01)$ & .10 \\
Reference height (per 0.1 mm greater) & $1.05(0.80-1.40)$ & .70 \\
RB outside normal limits & $0.98(0.95-1.40)$ & .26 \\
FSM outside normal limits & $2.44(1.47-4.06)$ & .001 \\
GPS global outside normal limits & $1.44(0.78-2.66)$ & .24 \\
MRA result outside normal limits & $2.29(1.19-4.40)$ & .013 \\
\hline
\end{tabular}

$\mathrm{C} / \mathrm{D}=$ cup-to-disc $; \mathrm{Cl}=$ confidence interval; CSA = crosssectional area; FSM = Frederick Mikelberg discriminant function; GPS = Glaucoma Probability Score; HR = hazard ratio; HRT = Heidelberg Retina Tomograph; HVC = height variation contour; MRA = Moorfields regression analysis; OAG = open-angle glaucoma; RB = Reinhard Burk discriminant function; RNFL = retinal nerve fiber layer.

One multivariate model was then run for each single HRT parameter to test the independent relationship of each HRT parameter with OAG (for a total of 11 models). Factors significantly predictive of the development of OAG in the multivariate model (without HRT disc area) included rim area, rim volume, $\mathrm{C} / \mathrm{D}$ area ratio, $\mathrm{C} / \mathrm{D}$ linear ratio, cup depth, cup shape measure, and FSM function outside normal limits. In this model, older age, higher IOP, and thinner CCT were also significantly predictive of the development of OAG (Table 4).

Factors significantly predictive of the development of OAG in the multivariate model (with HRT disc area as covariate) included cup area, rim area, cup volume, rim volume, C/D area ratio, C/D linear ratio, cup depth, maximum cup depth, cup shape measure, and FSM function outside normal limits. In this model, older age, higher IOP, and thinner CCT were also significantly predictive of the development of OAG (Table 5). MRA results outside normal limits were not included in this model, as MRA is based on the evaluation of rim area as a function of disc area. However, in a secondary analysis, which also included MRA, no statistically significant association was found between MRA and the development of glaucoma (HR 1.9; 95\% CI 0.9-4.0; $P=.091)$.
TABLE 4. Multivariate Hazard Ratios and 95\% Confidence Intervals for the Development of Open-Angle Glaucoma (Average of the Eyes), Including the Model Age, Baseline Intraocular Pressure, Central Corneal Thickness, and Pattern Standard Deviation

\begin{tabular}{lll}
\hline \multicolumn{1}{c}{ HRT Parameter } & HR (95\% Cl) & $P$ \\
\hline Cup area (per 0.3 $\mathrm{mm}^{2}$ greater) & $1.11(0.98-1.25)$ & .095 \\
Rim area (per 0.2 $\mathrm{mm}^{2}$ greater) & $0.78(0.63-0.95)$ & .014 \\
Cup volume (per $0.1 \mathrm{~mm}^{2}$ greater) & $1.16(0.99-1.36)$ & .07 \\
Rim volume (per 0.1 $\mathrm{mm}^{3}$ greater) & $0.80(0.65-0.99)$ & .041 \\
C/D area (per 0.1 greater) & $1.25(1.04-1.49)$ & .015 \\
C/D linear (per 0.1 greater) & $1.26(1.05-1.61)$ & .013 \\
Cup depth (per 0.1 mm greater) & $1.46(1.11-1.93)$ & .007 \\
Maximum cup depth & $1.15(1.00-1.32)$ & .052 \\
$\quad$ (per 0.1 mm greater) & & \\
Cup shape measure (per 0.1 greater) & $1.65(1.08-2.52)$ & .009 \\
FSM outside normal limits & $2.40(1.40-4.09)$ & .001 \\
GPS global outside normal limits & $0.93(0.48-1.80)$ & .82 \\
MRA result outside normal limits & $1.75(0.87-3.51)$ & .11 \\
Age (per 10 y older) & $1.41-1.58$ & $<.05$ \\
Baseline IOP (per 1 mm Hg higher) & $1.18-1.22$ & $<.05$ \\
CCT (per 40 $\mu \mathrm{m}$ thinner) & $1.51-1.59$ & $<.05$ \\
PSD (per 0.2 dB greater) & $1.04-1.19$ & $>.05$ \\
\hline
\end{tabular}

$\mathrm{CCT}=$ central corneal thickness; $\mathrm{C} / \mathrm{D}=$ cup-to-disc; $\mathrm{Cl}=$ confidence interval; FSM = Frederick Mikelberg discriminant function; GPS = Glaucoma Probability Score; HR = hazard ratio; HRT = Heidelberg Retina Tomograph; IOP = intraocular pressure; MRA = Moorfields regression analysis; $\mathrm{OAG}=$ openangle glaucoma; $P S D$ = pattern standard deviation.

The hazard ratios of all the covariates are reported as ranges among the various models. The $P$ value result was always $<.05$ or $>.05$.

The survival curves of the summary results of HRT analysis_-FSM, GPS global result, and MRA — show that the univariate cumulative probability of developing $\mathrm{OAG}$ within a time span of 5 years among subjects with an abnormal HRT summary result vs those with a normal HRT summary result at baseline was significantly higher in the case of an abnormal FSM $\left(\chi^{2}-\log\right.$ rank: 12.48; $P=.0004)$ and an abnormal MRA $\left(\chi^{2}-\log\right.$ rank: 6.33; $P=.0119)$, but not in the case of an abnormal GPS $\left(\chi^{2}\right.$ log rank: $1.34 ; P=.247$ ) (Figures $1-3$ ).

\section{DISCUSSION}

IT IS DEEMED IMPORTANT TO ASSESS THE PREDICTIVE (prognostic) factors for the development of OAG in order to identify the OHT patients at greater risk for future progression to OAG who may benefit from a closer follow-up evaluation and/or a prompt therapeutic approach.

The data collected from the EGPS HRT ancillary study and reported in this manuscript provide information about 
TABLE 5. Multivariate Hazard Ratios and 95\% Confidence Intervals for the Development of Open-Angle Glaucoma (Average of the Eyes), Including the Model Age, Baseline Intraocular Pressure, Central Corneal Thickness, Pattern Standard Deviation, and Heidelberg Retina Tomograph Disc Area

\begin{tabular}{lll}
\hline \multicolumn{1}{c}{ HRT Parameter } & HR (95\% Cl) & $P$ \\
\hline Cup area (per 0.3 $\mathrm{mm}^{2}$ greater) & $1.33(1.08-1.64)$ & .008 \\
Rim area (per 0.2 $\mathrm{mm}^{2}$ greater) & $0.75(0.61-0.93)$ & .008 \\
Cup volume (per $0.1 \mathrm{~mm}^{2}$ greater) & $1.30(1.05-1.61)$ & .017 \\
Rim volume (per 0.1 $\mathrm{mm}^{3}$ greater) & $0.80(0.65-0.99)$ & .041 \\
C/D area (per 0.1 greater) & $1.43(1.14-1.80)$ & .002 \\
C/D linear (per 0.1 greater) & $1.43(1.13-1.80)$ & .003 \\
Cup depth (per 0.1 mm greater) & $1.64(1.21-2.23)$ & .002 \\
Maximum cup depth & $1.18(1.01-1.36)$ & .031 \\
$\quad$ (per 0.1 mm greater) & & \\
Cup shape measure (per 0.1 greater) & $1.86(1.16-2.99)$ & .009 \\
FSM outside normal limits & $2.51(1.41-4.35)$ & .001 \\
GPS global outside normal limits & $0.98(0.47-2.03)$ & .95 \\
Age (per 10 y older) & $1.36-1.58$ & $<.05$ \\
Baseline IOP (per 1 mm Hg higher) & $1.18-1.22$ & $<.05$ \\
CCT (per 40 $\mu \mathrm{m}$ thinner) & $1.51-1.60$ & $<.05$ \\
PSD (per 0.2 dB greater) & $1.02-1.19$ & $>.05$ \\
HRT disc area (per 0.4 mm ${ }^{2}$ greater) & $0.32-1.34$ & $>.05$ \\
\hline
\end{tabular}

CCT = central corneal thickness; $\mathrm{C} / \mathrm{D}=$ cup-to-disc; $\mathrm{Cl}=$ confidence interval; FSM = Frederick Mikelberg discriminant function; GPS = Glaucoma Probability Score; $\mathrm{HR}$ = hazard ratio; HRT = Heidelberg Retina Tomograph; IOP = intraocular pressure; $\mathrm{OAG}=$ open-angle glaucoma; $\mathrm{PSD}=$ pattern standard deviation.

The hazard ratios of all the covariates are reported as ranges among the various models. The $P$ value result was always $<.05$ or $>.05$.

the HRT-based predictive factors for the development of OAG within 5 years in OHT patients.

In the EGPS, after adjusting for the treatment arm, age, PSD, CCT, and HRT disc area, the HRT measurements that significantly predicted the development of OAG over a 5-year period were cup area, cup volume, C/D area, C/D linear, cup depth, maximum cup depth, cup shape measure, rim area, rim volume, and the FSM multidiscriminant function.

The OHTS is the first multicenter, long-term clinical trial on OHT patients to have evaluated their data for HRT. based predictive factors in a subset of the original sample. Two different papers have reported the OHTS HRT predictive value for the development of POAG: 1 within 5 years ${ }^{35}$ and another within 12 years. ${ }^{36}$ If the predictive factors observed in the EGPS and those observed in the OHTS are compared, it is quite clear that the 2 studies had several consistent results (Table 6). Some differences in terms of the absolute values of the HRs may be explained by some protocol differences between the 2 studies, by population differences, and by differences in the follow-up time.

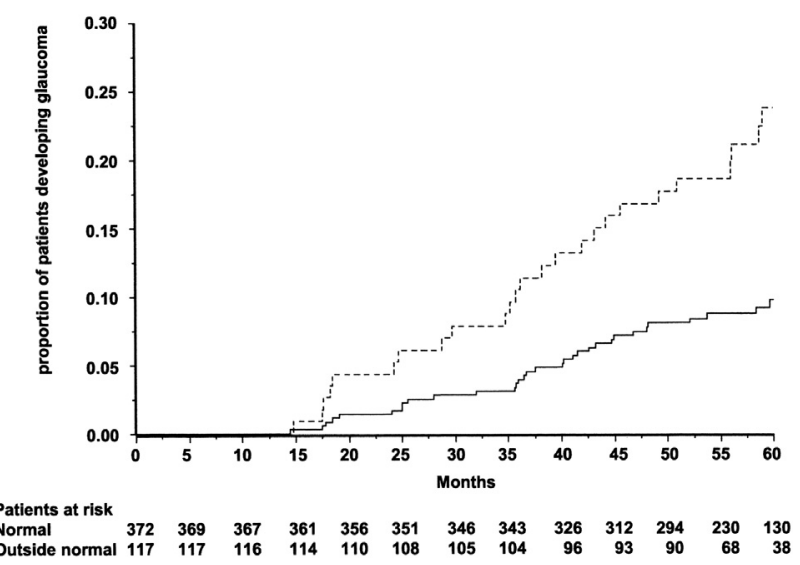

FIGURE 1. Kaplan-Meier plot of the univariate cumulative probability of developing open-angle glaucoma by the Frederick Mikelberg discriminant function result outside normal limits (dashed lines) compared with those not outside normal limits (bold line). The difference is statistically significant $\left(\chi^{2}-\log \right.$ rank: $12.48 ; P=.0004)$.

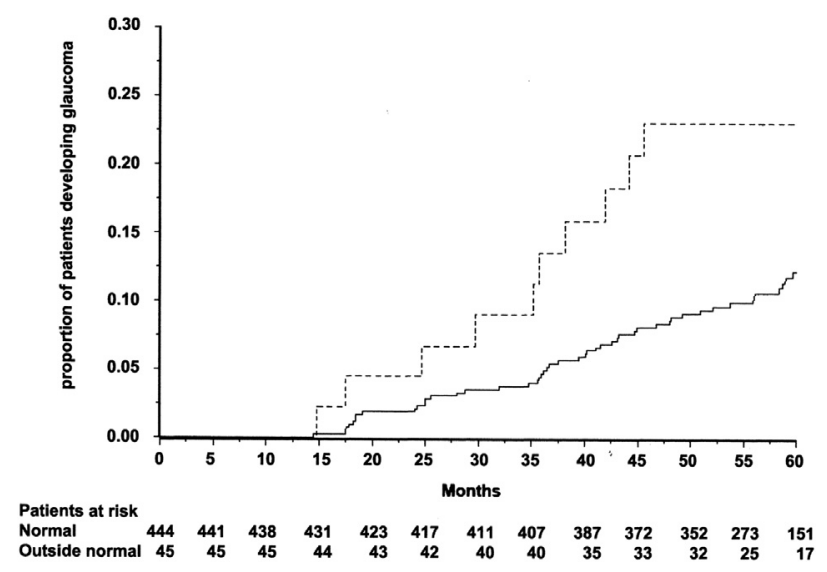

FIGURE 2. Kaplan-Meier plot of the univariate cumulative probability of developing open-angle glaucoma by the Moorfields regression analysis result outside normal limits (dashed lines) compared with those not outside normal limits (bold line). The difference is statistically significant $\left(\chi^{2}-\log\right.$ rank: 6.33; $P=.0119)$.

As a matter of fact, most of the stereometric variables were significantly predictive for the development of OAG in OHTS. The only difference between the EGPS and the OHTS stereometric variables results was related to cup shape measure that our study confirmed as being a statistically and clinically significant marker for the development of OAG, whereas the OHTS did not. Cup shape measure has been identified from several studies as one of the most reliable stereometric HRT measurement for the early identification of glaucoma-related abnormality of the optic disc. ${ }^{20,48-53}$ Cup shape measure, previously known as "third momentum," describes the shape of the 


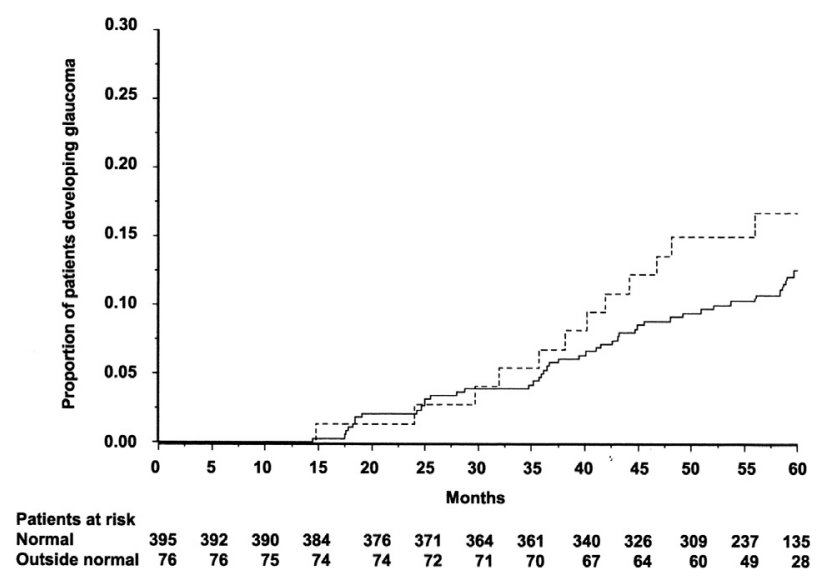

FIGURE 3. Kaplan-Meier plot of the univariate cumulative probability of developing open-angle glaucoma by the Glaucoma Probability Score result outside normal limits (dashed lines) compared with those not outside normal limits (bold line). The difference is not statistically significant $\left(\chi^{2}-\log\right.$ rank: 1.34; $P=.247$ ).

optic disc cupping and is inherent in the FSM and RB discriminant formulas.

Interestingly, and in total agreement with the OHTS, no "RNFL"-based HRT measurement was predictive of OAG development. RNFL thickness and RNFL cross-sectional area have always been improperly referred to as RNFL parameters. These measurements refer to the position of the reference plane along the "z" axis, which is, by default, localized $50 \mu \mathrm{m}$ below the surface at the papillomacular bundle localized along the contour line ${ }^{54}$ For this reason, whatever is above the reference plane and along the contour line cannot reflect the anatomic region of the retina known as "RNFL." Although the RNFL becomes thinner in glaucomatous eyes, it is thus conceivable that the "surrogate measurement of RNFL" performed by the HRT is unable to properly identify its glaucomatous thinning. HVC is instead the measure of height variability of the topographic surface along the contour line. It should somewhat refer to the modulation of RNFL height along the optic disc border (identified by the contour line). Apparently, the same criticisms referring to the RNFL-related HRT parameters may hold true for HVC.

The rim- and cup-related parameters have been indicated as good markers for early glaucoma in the vast majority of the HRT-related investigations. ${ }^{18-21,23,27,29,35,36,49-52}$ In our study, however, cup area was a significant predictor for OAG development only when the multivariate analysis included HRT disc area in the model. Rim and cup areas are significantly correlated with disc area in normal eyes, both in morphometric studies ${ }^{44,45}$ and in HRT studies. ${ }^{46,47,55-58}$ This suggests that both cup area and rim area should increase their predictive value for the development of OAG by taking disc area into account.
This hypothesis has been clearly confirmed for cup area but not for rim area in our study, suggesting that rim area or rim-related variables may be more easily handled by the ophthalmologist in order to better estimate the individual risk profile of the OHT patient.

The multidiscriminant functions $\mathrm{FSM}^{16}$ and $\mathrm{RB},{ }^{25}$ as well as the classification systems such as the $\mathrm{MRA}^{21}$ and the GPS, ${ }^{24}$ have been developed in order to achieve an accurate and simple clinical identification of abnormal (glaucomatous) discs. Several cross-sectional studies have investigated their diagnostic ability, often reporting good results. ${ }^{16,19,21,24,25,27,59}$ Other longitudinal studies have reported a significant predictability for the development of POAG in the case of MRA, ${ }^{60,61}$ but not for GPS. ${ }^{60}$ In the OHTS both FSM and MRA were significantly predictive for the development of POAG at 5 years, ${ }^{35}$ whereas MRA and GPS were significantly predictive for the development of POAG at 12 years. ${ }^{36}$ In the EGPS, with a followup of 5 years, FSM was significantly predictive for the development of OAG, whereas MRA and GPS were not in the multivariate models including age, baseline IOP, CCT, and PSD (with and without HRT disc area). However, MRA was significantly predictive in univariate analysis, and the survival curve showed that the univariate cumulative probability of developing $\mathrm{OAG}$ within a time span of 5 years among subjects with an abnormal MRA result vs those with a normal MRA result at baseline was significantly higher $\left(\chi^{2}-\log\right.$ rank test: 6.33; $\left.P=.0119\right)$ (Figure 2). Moreover, if we focus on the graphs of the survival curves of MRA and GPS reported in this study (on a time span of 5 years) (Figures 2 and 3 ) and on those published by the OHTS (on a time span of 12 years), ${ }^{36}$ and compare the graphs at the time point of 60 months, it seems quite clear that the separation of the 2 cumulative curves in the 2 graphs of the 2 studies are very similar in the case of both MRA and GPS. We think that at the time point of 60 months the results for MRA and GPS in the OHTS and EGPS are quite the same (ie, GPS was not significantly predictive of the development of POAG also in the OHTS). Although this hypothesis cannot be mathematically confirmed by us, it cannot be ruled out.

It is always difficult to compare results from different studies, given differences in the selection of the sample, in the sample size, in the definition of the disease, ${ }^{62}$ in the methodology, and in the follow-up (in case of longitudinal studies). In the case of HRT studies, other sources of variability may add to the results, such as the quality of the images, the definition of the contour line (which is manually drawn), and the influence of disc size on MRA, GPS, RB, and FSM discriminant functions. ${ }^{46,47,63,64}$ The EGPS is the largest cohort of OHT white patients studied by means of HRT and followed up for 5 years. The definition of $O A G$ is based on standardized criteria, established before the start of the trial. ${ }^{39}$ Assessment of image quality was performed by means of the automatic quality control of the software according to the recommended 
TABLE 6. Multivariate Hazard Ratios and 95\% Confidence Intervals for the Development of Open-Angle Glaucoma (Average of the Eyes), Including the Model Age, Baseline Intraocular Pressure, Central Corneal Thickness, Pattern Standard Deviation, and Heidelberg Retina Tomograph Disc Area, in the European Glaucoma Prevention Study and Hazard Ratios Reported by the Ocular Hypertensive Treatment Study

\begin{tabular}{|c|c|c|c|}
\hline HRT Parameter & $\begin{array}{l}\text { EGPS } 5 \text { Years } \\
\text { HR }(95 \% \mathrm{Cl})\end{array}$ & 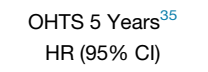 & 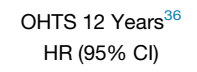 \\
\hline Cup area (per $0.3 \mathrm{~mm}^{2}$ greater) & $1.33(1.08-1.64)$ & $1.21(0.96-1.53)$ & $1.47(1.21-1.79)$ \\
\hline Rim area (per $0.2 \mathrm{~mm}^{2}$ greater) & $0.75(0.61-0.93)$ & $0.57(0.42-0.78)$ & $0.46(0.36-0.59)$ \\
\hline Cup volume (per $0.1 \mathrm{~mm}^{2}$ greater) & $1.30(1.05-1.61)$ & $1.20(1.01-1.43)$ & $1.40(1.23-1.60)$ \\
\hline Rim volume (per $0.1 \mathrm{~mm}^{3}$ greater) & $0.80(0.65-0.99)$ & $0.65(0.47-0.91)$ & $0.53(0.39-0.72)$ \\
\hline$C / D$ area (per 0.1 greater) & $1.43(1.14-1.80)$ & $1.25(1.02-1.53)$ & $1.64(1.36-1.96)$ \\
\hline C/D linear (per 0.1 greater) & $1.43(1.13-1.80)$ & - & $1.71(1.36-2.15)$ \\
\hline Cup depth (per $0.1 \mathrm{~mm}$ greater) & $1.64(1.21-2.23)$ & $1.60(1.15-2.22)$ & $1.93(1.50-2.49)$ \\
\hline Maximum cup depth (per $0.1 \mathrm{~mm}$ greater) & $1.18(1.01-1.36)$ & - & - \\
\hline Cup shape measure (per 0.1 greater) & $1.86(1.16-2.99)$ & $1.02(0.62-1.67)$ & $1.33(0.94-1.88)$ \\
\hline FSM outside normal limits & $2.51(1.41-4.35)$ & $2.54(1.31-4.90)$ & - \\
\hline GPS global outside normal limits & $0.98(0.47-2.03)$ & - & $3.51(1.83-6.73)$ \\
\hline MRA result outside normal limits & $1.75(0.87-3.51)$ & $2.39(1.02-5.62)$ & $3.90(2.09-7.28)$ \\
\hline
\end{tabular}

TABLE 7. Multivariate Hazard Ratios and 95\% Confidence Intervals of the Clinical Predictive Factors for the

Development of Open-Angle Glaucoma (Average of the Eyes) Reported in the Original European Glaucoma Prevention Study ${ }^{12}$ and Observed in the Present Study

\begin{tabular}{lcc}
\hline \multicolumn{1}{c}{ Parameter } & $\begin{array}{c}\text { EGPS } \\
\text { HR }(95 \% \mathrm{Cl})\end{array}$ & $\begin{array}{c}\text { EGPS HRT Substudy } \\
\text { HR }(95 \% \mathrm{Cl})\end{array}$ \\
\hline Age (per decade) & $1.32(1.04-1.69)$ & $1.52(1.05-2.19)$ \\
IOP, baseline (per mm Hg) & $1.07(0.94-1.22)$ & $1.10(0.93-1.30)$ \\
CCT (per 40 $\mu \mathrm{m}$ thinner) & $1.32(1.05-1.67)$ & $1.71(1.21-2.41)$ \\
Vertical C/D ratio & $1.34(1.14-1.58)$ & $1.88(1.38-2.58)$ \\
$\quad$ (per 0.1 larger) & & \\
PSD (per 0.2 dB greater) & $1.66(1.15-2.38)$ & $1.53(0.92-2.56)$ \\
\hline
\end{tabular}

$\mathrm{CCT}=$ central corneal thickness; $\mathrm{C} / \mathrm{D}=$ cup-to-disc; $\mathrm{Cl}=$ confidence interval; EGPS = European Glaucoma Prevention Study; HR = hazard ratio; IOP = intraocular pressure; OAG = open-angle glaucoma; PSD = pattern standard deviation.

criteria (to reject images): images whose standard deviation was greater than $50 \mu \mathrm{m}$ were automatically excluded. ${ }^{37}$ The optic disc margin was outlined by 1 experienced examiner and each outline of the optic disc was reviewed by a second experienced examiner (with differences of opinion resolved by consensus). ${ }^{37} \mathrm{~A}$ second independent image quality control step was performed at the OHTS CSLO Reading Center, using the same review criteria that had been used for the OHTS CSLO Ancillary Study. ${ }^{34-36}$ Differently from the OHTS, which included data for both eyes of the participant in the model, and used the method of Lee and associates ${ }^{65}$ to adjust for the correlation of the 2 eyes from the same participant, the EGPS averaged the measurements of the 2 eyes in order to be consistent with the methodology applied in the assessment of the baseline clinical predictive factors, ${ }^{12}$ and flagged as "outside the normal limits" all the cases whose classification was outside in 1 eye and not outside normal limits in the fellow eye. Moreover, the mean HRT disc size of the EGPS sample is slightly larger than the mean HRT disc area reported in the OHTS. ${ }^{35}$ These discrepancies may have contributed to the slightly different results reported for some variables in the 2 studies.

This study may have some limitations as well, and both the possible inadequate representativeness of the original EGPS population and technical concerns related to HRT protocol have been previously addressed and discussed. ${ }^{37}$ To better address the issue of inadequate representativeness, it is of note that the HRs of the predictive factors previously reported in the EGPS (age, IOP, CCT, vertical C/D ratio, and PSD $)^{12}$ and those observed in this subset are similar, but tend to indicate that this subset of the original EGPS population may be at higher risk to develop OAG (Table 7).

The results of the EGPS HRT substudy may be clinically relevant as they support the use of HRT in the clinical practice, as a useful clinical tool to perform optic disc topographic measurements in OHT patients who may have a high risk to develop OAG. Although it has been consistently shown that an accurate clinical evaluation of the optic disc with an estimate of the $\mathrm{C} / \mathrm{D}$ ratio may be sufficient to help the clinician in estimating the individual OHT patient's risk to develop OAG within the next 5 years, the 
results of this study, in agreement with those of the OHTS, seem to indicate that several HRT parameters/classification systems are statistically and clinically significant prognostic factors for the development of OAG. This does not mean that HRT replaces clinical judgment of the optic disc. Instead it supports the implementation of HRT in the daily clinical practice, as it has been recently shown that its diagnostic ability outperforms the diagnostic ability of general ophthalmologists. ${ }^{66}$

Lastly, the results of the EGPS HRT substudy will contribute to the ongoing OHTS-EGPS HRT collaborative study with the aim of providing a further refinement of the risk model previously established by the collaboration of the 2 studies. ${ }^{13,14}$

ALL AUTHORS HAVE COMPLETED AND SUBMITTED THE ICMJE FORM FOR DISCLOSURE OF POTENTIAL CONFLICTS OF INTEREST and report the following financial disclosures: S.M.: lecture fees from Merck, Alcon, Allergan, SIFI Italia, Omikron Italia, Pfizer; V.T.: payments for development of educational presentations from Roche; I.F.: consultancy fee from Bayer; N.P.: board membership fees from Alcon, Allergan, Ivantis, and Santen; consultancy fee from Ivantis; lecture fees from Alcon, Santen, Allergan, and Merck. The European Glaucoma Prevention Study is supported by The European Commission (BIOMED II program, contract BMH4-CT-96-1598) and Merck \&Co, Inc (Whitehouse Station, New Jersey, USA). Merck \& Co provided the study medications. Heidelberg Engineering provided the HRT devices at no costs, as well as travel and lecture support. The sponsors and the funding organizations had no role in the design or conduct of this research. Contributions of authors: design and conduct of the study (S.M., T.Z., V.T., J.C.-V., N.P.); collection, management, analysis, and interpretation of the data (S.M., T.Z., E.H., E.R., I.F., D.P., S.A.); preparation, review, or approval of the manuscript (S.M., T.Z., E.R., I.F., N.P.).

\section{REFERENCES}

1. Anderson DR. Glaucoma: the damage caused by pressure. XLVI Edward Jackson memorial lecture. Am J Ophthalmol 1989;108(5):485-495.

2. American Academy of Ophthalmology. Primary Open-angle Glaucoma. Preferred Practice Pattern. San Francisco: American Academy of Ophthalmology; 1992.

3. Sommer A, Tielsch JM, Katz J, et al. Relationship between intraocular pressure and primary open angle glaucoma among white and black Americans. The Baltimore Eye Survey. Arch Ophthalmol 1991;109(8):1090-1095.

4. Tielsch JM, Katz J, Sommer A, et al. Hypertension, perfusion pressure, and primary open angle glaucoma. A populationbased assessment. Arch Ophthalmol 1995;113(2):216-221.

5. Leske MC, Connell AM, Wu SY, et al. Risk factors for openangle glaucoma. The Barbados Eye Study. Arch Ophthalmol 1995;113(7):918-924.

6. Leske MC, Connell AMS, Wu S-Y, et al. Incidence of open angle glaucoma. The Barbados Eye Study. Arch Ophthalmol 2001;119(1):89-95.

7. Tielsch JM, Katz J, Sommer A, et al. Family history and risk of primary open angle glaucoma. The Baltimore Eye Survey. Arch Ophthalmol 1994;112(1):69-73.

8. Bonomi L, Marchini G, Marraffa M, et al. Vascular risk factors for primary open angle glaucoma: the Egna-Neumarkt Study. Ophthalmology 2000;107(7):1287-1293.

9. Leske MC, Wu S-Y, Nemesure B, et al. Incident open angle glaucoma and blood pressure. Arch Ophthalmol 2002;120(7): 954-959.

10. Kass MA, Heuer DK, Higginbotham EJ, et al. The Ocular Hypertension Treatment Study. A randomized trial determines that topical hypotensive medication delays or prevents the onset of Primary Open-Angle Glaucoma. Arch Ophthalmol 2002;120(6):701-713.

11. Gordon MO, Beiser JA, Brandt JD, et al. The Ocular Hypertension Treatment Study. Baseline factors that predict the onset of primary open-angle glaucoma. Arch Ophthalmol 2002;120(6):714-720.
12. Miglior S, Pfeiffer N, Torri V, et al; for The European Glaucoma Prevention Study Group. Predictive factors for open angle glaucoma among patients with ocular hypertension in the European Glaucoma Prevention Study. Ophthalmology 2007;114(1):3-9.

13. Gordon M, Torri V, Miglior S, et al; for The Ocular Hypertension Treatment Study Group and the European Glaucoma Prevention Study Group. A validated prediction model for the development of primary open angle glaucoma in individuals with ocular hypertension. Ophthalmology 2007;114(1):10-19.

14. The Ocular Hypertension Treatment Study Group, the European Glaucoma Prevention Study Group. The accuracy and clinical application of predictive models for primary openangle glaucoma in ocular hypertensive individuals. Ophthalmology 2008;115(11):2030-2036.

15. Kruse FE, Burk RO, Volcker HE, et al. Reproducibility of topographic measurements of the optic nerve head with laser tomographic scanning. Ophthalmology 1989;96(9):1320-1324.

16. Mikelberg FS, Parfitt CM, Swindale NV, et al. Ability of the Heidelberg Retina Tomograph to detect early glaucomatous visual field loss. J Glancoma 1995;4(4):242-247.

17. Asawaphureekorn S, Zangwill L, Weinreb RN. Rankedsegment distribution curve for interpretation of optic nerve topography. J Glaucoma 1996;5(2):79-90.

18. Bartz-Schmidt KU, Sengersdorf A, Esser P, et al. The cumulative normalised rim/disc area ratio curve. Graefes Arch Clin Exp Ophthalmol 1996;234(4):227-231.

19. Iester M, Broadway DC, Mikelberg FS, Drance SM. A comparison of healthy, ocular hypertensive, and glaucomatous optic disc topographic parameters. J Glancoma 1997;6(6):363-370.

20. Bathija R, Zangwill L, Berry CC, et al. Detection of early glaucomatous structural damage with confocal scanning laser tomography. J Glaucoma 1998;7(2):121-127.

21. Wollstein G, Garway-Heath DF, Hitchings RA. Identification of early glaucoma cases with the scanning laser ophthalmoscope. Ophthalmology 1998;105(8):1557-1563.

22. Caprioli J, Park HJ, Ugurlu S, Hoffman D. Slope of the peripapillary nerve fiber layer surface in glaucoma. Invest Ophthalmol Vis Sci 1998;39(12):2321-2328. 
23. Kamal DS, Viswanathan AC, Garway-Heath DF, et al. Detection of optic disc change with the Heidelberg retina tomograph before confirmed visual field change in ocular hypertensives converting to early glaucoma. Br J Ophthalmol 1999;83(3):290-294.

24. Swindale NV, Stjepanovic G, Chin A, Mikelberg FS. Automated analysis of normal and glaucomatous optic nerve head topography images. Invest Ophthalmol Vis Sci 2000;41(7): 1730-1742.

25. Burk ROW, Noack H, Rohrschneider K, Volcker HE. Prediction of glaucomatous visual field defects by reference plane independent three-dimensional optic nerve head parameters. In: Wall M, Wild JM, eds. Perimetry Update 1998/1999. The Hague, The Netherlands: Kugler Publications; 1999:463-474.

26. Miglior S, Casula M, Guareschi M, et al. Clinical ability of Heidelberg Retinal Tomograph examination in the detection of glaucomatous visual field changes. Ophthalmology 2001; 108(9):1621-1627.

27. Miglior S, Guareschi M, Albe' E, et al. Detection of glaucomatous visual field changes using the Moorfields Regression Analysis of the Heidelberg Retinal Topography. Am J Ophthalmol 2003;136(1):26-33.

28. Hoffmann EM, Boden C, Zangwill LM, et al. Inter-eye spatial relationship of abnormal neuroretinal rim locations in glaucoma patients from the diagnostic innovations in glaucoma study. Am J Ophthalmol 2007;143(5):781-787.

29. De Leon-Ortega JE, Sakata LM, Monheit BE, et al. Comparison of diagnostic accuracy of Heidelberg Retina Tomograph II and Heidelberg Retina Tomograph 3 to discriminate glaucomatous and non-glaucomatous eyes. Am J Ophthalmol 2007; 144(4):525-532.

30. Ferreras A, Pablo LE, Larosa JM, et al. Discriminating between normal and glaucoma-damaged eyes with the Heidelberg Retina Tomograph 3. Ophthalmology 2008;115(5): 775-781.

31. Iester M, Perdicchi A, Capris E, et al. Comparison between discriminant analysis models and "glaucoma probability score" for the detection of glaucomatous optic nerve head changes. J Glaucoma 2008;17(7):535-540.

32. Medeiros FA, Vizzeri G, Zangwill LM, et al. Comparison of retinal nerve fiber layer and optic disc imaging for diagnosing glaucoma in patients suspected of having the disease. Ophthalmology 2008;115(8):1340-1346.

33. Strouthidis NG, Demirel S, Asaoka R, et al. The Heidelberg retina tomograph Glaucoma Probability Score: reproducibility and measurement of progression. Ophthalmology 2010;117(4):724-729.

34. Zangwill LM, Weinreb RN, Berry CC, et al. The confocal scanning laser ophthalmoscopy ancillary study to the ocular hypertension treatment study: study design and baseline factors. Am J Ophthalmol 2004;137(2):219-227.

35. Zangwill LM, Weinreb RN, Beiser J, et al. Baseline topographic optic disc measurements are associated with the development of primary open-angle glaucoma. The confocal scanning laser ophthalmoscopy ancillary study to the Ocular Hypertension Treatment Study. Arch Ophthalmol 2005; 123(9):1188-1197.

36. Weinreb RN, Zangwill LM, Jain S, et al. Predicting the onset of glaucoma: the Confocal Scanning Laser Ophthalmoscopy Ancillary Study to the Ocular Hypertension Treatment Study. Ophthalmology 2010;117(9):1674-1683.
37. Hoffmann EM, Miglior S, Zeyen T, et al. The Heidelberg retina tomograph ancillary study to the European glaucoma prevention study: study design and baseline factors. Acta Ophthalmol 2013;91(8):612-619.

38. The European Glaucoma Prevention Study Group. Results of the European Glaucoma Prevention Study. Ophthalmology 2005;112(3):366-375.

39. The European Glaucoma Prevention Study Group. The European Glaucoma Prevention Study. Design and baseline description of the participants. Ophthalmology 2002;109(9): $1612-1621$.

40. The European Glaucoma Prevention Study Group. Reproducibility of evaluation of optic disc change for glaucoma with stereo optic disc photographs. Ophthalmology 2003; $110(2): 340-344$.

41. Zeyen T, Roche M, Brigatti L, Caprioli J. Formulas for conversion between Octopus and Humphrey threshold values and indices. Graefes Arch Clin Exp Ophthalmol 1995; 233(10):627-634.

42. The European Glaucoma Prevention Study Group. Central corneal thickness in the European Glaucoma Prevention Study. Ophthalmology 2007;114(3):454-459.

43. Brandt JD, Beiser JA, Kass MA, et al. Central corneal thickness in the Ocular Hypertension Treatment Study (OHTS). Ophthalmology 2001;108(10):1779-1788.

44. Britton RJ, Drance SM, Schulzer M, et al. The area of neuroretinal rim of the optic nerve in normal eyes. Am J Ophthalmol 1987;103(4):497-504.

45. Jonas JB, Gusek GC, Naumann GOH. Optic disc, cup and neuroretinal rim size, configuration and correlation in normal eyes. Invest Ophthalmol Vis Sci 1988;29(7):1151-1158.

46. Iester M, Mikelberg FS, Drance SM. The effect of optic disc size on diagnostic precision with the Heidelberg retina tomograph. Ophthalmology 1997;104(3):545-548.

47. Medeiros FA, Zangwill LM, Bowd C, et al. Influence of disease severity and optic disc size on the diagnostic performance of imaging instruments in glaucoma. Invest Ophthalmol Vis Sci 2006;47(3):1008-1015.

48. Brigatti L, Caprioli J. Correlation of visual field with scanning confocal laser optic disc measurements in glaucoma. Arch Ophthalmol 1995;113(9):1191-1194.

49. Uchida H, Brigatti L, Caprioli J. Detection of structural damage from glaucoma with confocal laser image analysis. Invest Ophthalmol Vis Sci 1996;37(12):2393-2401.

50. Iester M, Mikelberg FS, Courtright P, Drance SM. Correlation between the visual field indices and Heidelberg retina tomograph parameters. J Glaucoma 1997;6(2):78-82.

51. Iester M, Mikelberg FS, Swindale NV, Drance SM. ROC analysis of Heidelberg Retina Tomograph optic disc shape measures in glaucoma. Can J Ophthalmol 1997;32(6): 382-388.

52. Mistlberger A, Liebmann JM, Greenfield DS, et al. Heidelberg retina tomography and optical coherence tomography in normal, ocular-hypertensive, and glaucomatous eyes. Ophthalmology 1999;106(10):2027-2032.

53. Philippin H, Unsoeld A, Maier P, et al. Ten-year results: detection of long-term progressive optic disc changes with confocal laser tomography. Graefes Arch Clin Exp Ophthalmol 2006;244(4):460-464.

54. Burk RO, Vihanninjoki K, Bartke T, et al. Development of the standard reference plane for the Heidelberg retina 
tomograph. Graefes Arch Clin Exp Ophthalmol 2000;238(5): 375-384.

55. Bowd C, Zangwill LM, Blumenthal EZ, et al. Imaging of the optic disc and retinal nerve fiber layer: the effects of age, optic disc area, refractive error, and gender. J Opt Soc Am A Opt Image Sci Vis 2002;19(1):197-207.

56. Vernon SA, Hawker MJ, Ainsworth G, et al. Laser scanning tomography of the optic nerve head in a normal elderly population: the Bridlington eye assessment project. Invest Ophthalmol Vis Sci 2005;46(8):2823-2828.

57. Uchida H, Yamamoto T, Araie M, et al. Topographic characteristics of the optic nerve head measured with scanning laser tomography in normal Japanese subjects. Jpn J Ophthalmol 2005;49(6):469-476.

58. Sawada Y, Ishikawa M, Sato N, Yoshitomi T. Optic nerve head morphology assessed by laser scanning tomography in normal Japanese subjects. J Glaucoma 2011;20(7):445-451.

59. Yip LW, Mikelberg FS. A comparison of the glaucoma probability score to earlier heidelberg retina tomograph data analysis tools in classifying normal and glaucoma patients. J Glaucoma 2008;17(7):513-516.

60. Strouthidis NG, Gardiner SK, Owen VM, et al. Predicting progression to glaucoma in ocular hypertensive patients. J Glaucoma 2010;19(5):304-309.
61. Larrosa JM, Polo V, Ferreras A, et al. Predictive value of confocal scanning laser for the onset of visual field loss in glaucoma suspects. Ophthalmology 2012;119(8):1558-1562.

62. Miglior S, Guareschi M, Romanazzi F, et al. The impact of definition of primary open-angle glaucoma on the crosssectional assessment of diagnostic validity of Heidelberg retinal tomography. Am J Ophthalmol 2005;139(5):878-887.

63. Zangwill LM, Jain S, Racette L, et al. The effect of disc size and severity of disease on the diagnostic accuracy of the Heidelberg Retina Tomograph Glaucoma Probability Score. Invest Ophthalmol Vis Sci 2007;48(6):2653-2660.

64. Oddone F, Centofanti M, Tanga L, et al. Influence of disc size on optic nerve head versus retinal nerve fiber layer assessment for diagnosing glaucoma. Ophthalmology 2011;118(7): 1340-1347.

65. Lee EW, Wei LJ, Amato D. Cox-type regression analysis for large numbers of small groups of correlated failure time observations. In: Klein JP, Goel PK, eds. Survival Analysis, State of the Art. Dordrecht, The Netherlands: Kluwer Academic; 1992:237-247.

66. Reus NJ, Lemij HG, Garway-Heath DF, et al. Clinical assessment of stereoscopic optic disc photographs for glaucoma: the European Optic Disc Assessment Trial. Ophthalmology 2010; 117(4):717-723. 


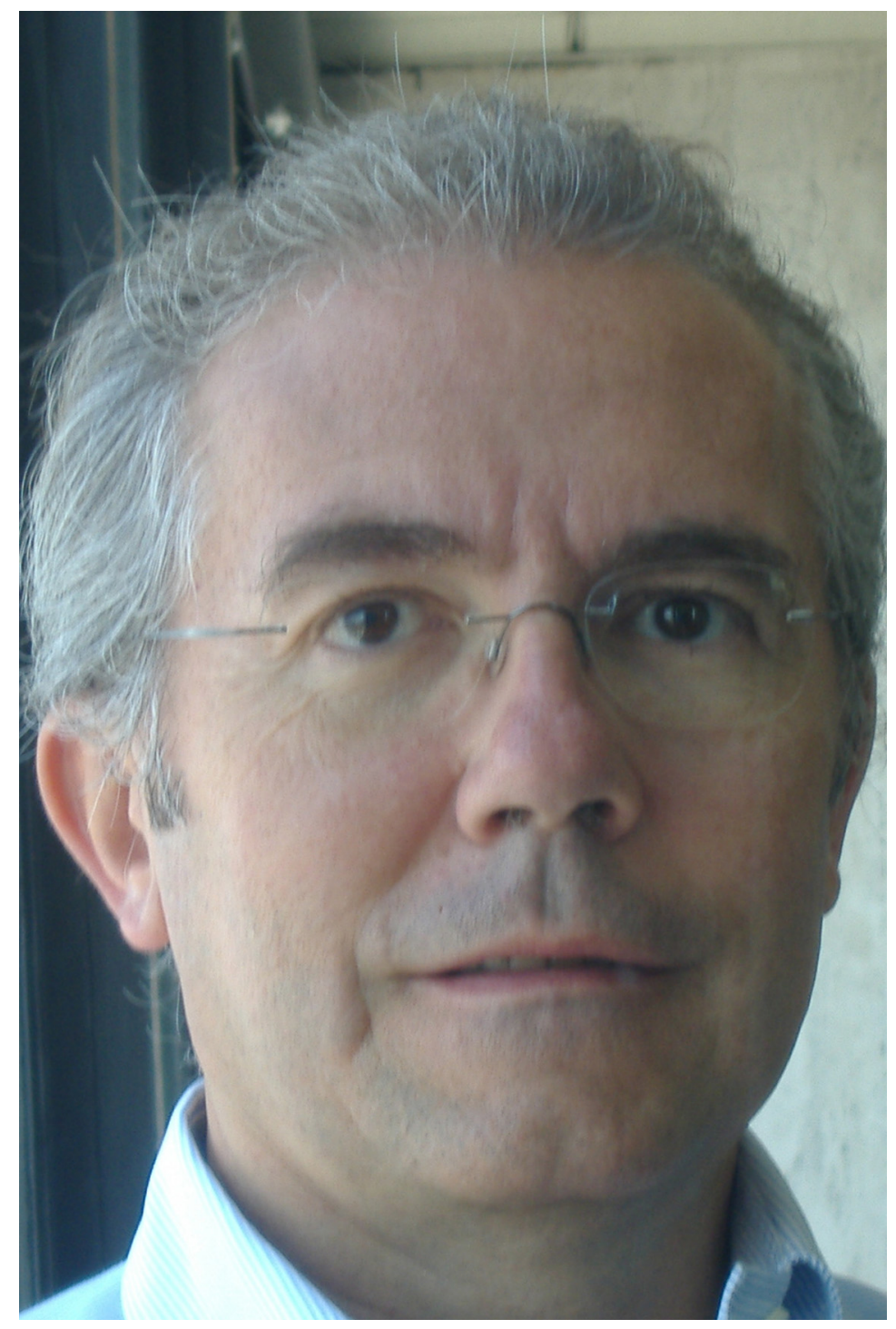

Biosketch

Stefano Miglior is Professor of Ophthalmology at the University Milano Bicocca, and Chair of the Ophthalmic Department of the Policlinico di Monza (MI), Italy. He is author of several papers on the diagnosis of glaucoma and imaging of the disc and Retinal Nerve Fiber Layer published on major international journals. He serves as reviewer for all of the major international journals. Stefano Miglior is the Principal Investigator of the European Glaucoma Prevention Study. 